\title{
Hierarchical Exploration of Volumes Using Multilevel Segmentation of the Intensity-Gradient Histograms
}

\author{
Cheuk Yiu Ip, Amitabh Varshney, Fellow, IEEE, and Joseph JaJa, Fellow, IEEE
}

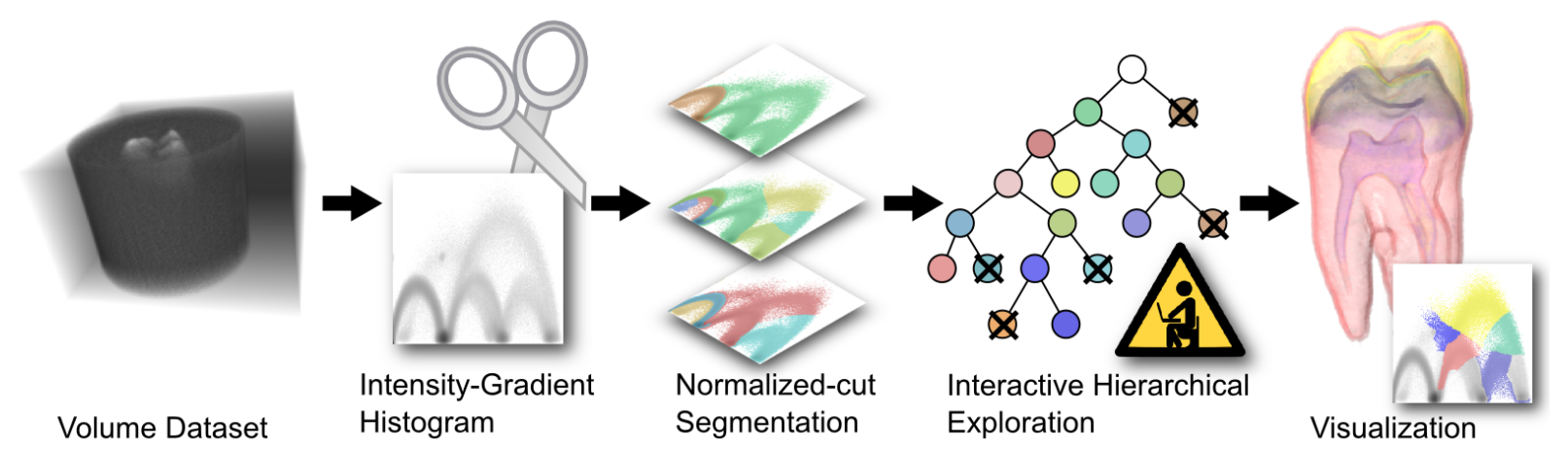

Fig. 1. We explore a volume dataset with an intensity-gradient histogram segmentation hierarchy. 1. We compute a $2 \mathrm{D}$ intensitygradient histogram from a volume dataset. 2 . We mimic the user visual search of shapes in the histogram by recursively segmenting the histogram image using normalized cuts. 3. We construct a multi-resolution hierarchy for interactive exploration. 4. Users traverse this hierarchy to discover features in the volume data and compose meaningful visualizations.

\begin{abstract}
Visual exploration of volumetric datasets to discover the embedded features and spatial structures is a challenging and tedious task. In this paper we present a semi-automatic approach to this problem that works by visually segmenting the intensitygradient 2D histogram of a volumetric dataset into an exploration hierarchy. Our approach mimics user exploration behavior by analyzing the histogram with the normalized-cut multilevel segmentation technique. Unlike previous work in this area, our technique segments the histogram into a reasonable set of intuitive components that are mutually exclusive and collectively exhaustive. We use information-theoretic measures of the volumetric data segments to guide the exploration. This provides a data-driven coarse-to-fine hierarchy for a user to interactively navigate the volume in a meaningful manner.
\end{abstract}

Index Terms-Volume exploration, volume classification, normalized cut, Information-guided exploration.

\section{INTRODUCTION}

Identifying and visualizing meaningful features in large volume datasets remains a significant challenge [20]. Visualization of the features that you know are in the data is hard. It is even harder to find the features that you do not know are there. While we have made significant strides in building up a substantial body of knowledge over the last two decades in direct volume rendering, much of these advances have addressed issues surrounding how to depict the data; what to depict remains an important problem. We seek a visualization approach that highlights meaningful information, guides users to explore, and allows the users to associate high-level knowledge with low-level raw data. We carry this out by visually segmenting the intensity-gradient histogram of a volumetric dataset into a hierarchy for exploration.

We first outline the three most important components of the data exploration challenge facing us today.

Information Challenge: Detecting and displaying meaningful features, trends, and anamolies in data is an important challenge in visualization. This is becoming an increasingly significant challenge as our ability to acquire data is surpassing our ability to meaningfully analyze it. In this paper we seek a way to locate and visualize data

- The authors are with the Institute for Advanced Computer Studies, University of Maryland, College Park. E-mail:

\{ipcy,varshney\}@cs.umd.edu, joseph@umiacs.umd.edu.

Manuscript received 31 March 2012; accepted 1 August 2012; posted online 14 October 2012; mailed on 5 October 2012.

For information on obtaining reprints of this article, please send

e-mail to:tvcg@computer.org. with the highest information content using simple statistically-based information-theoretic measures.

Completeness Challenge: Exhaustive data exploration is a tedious and time-intensive exercise and yet is important to ensure that we do not overlook any important features in the data. We need mechanisms that facilitate a complete data exploration. In this paper we show how to construct an exploration hierarchy to accomplish this goal using a top-down subdivision strategy to cover the entire feature space of a volume dataset.

Semantic Challenge: Current computational approaches may identify potentially informative regions by using low-level attributes such as statistics of data, the derivatives of the scalar field, or even embed the data into its own principal dimensions or manifold. However semantically driven navigation of the data is still a task that is designated for the user. We facilitate addressing this challenge by providing an intuitive and interactive volume exploration interface that is based on natural groupings that are often seen to be semantically related.

In practice, users manually search for regions of interest by inspecting different areas of a feature space. Popular exploration subspaces for such a feature space include 1D density and 2D intensitygradient. Histograms are often used to aid this search and have been implemented in several popular visualization packages, such as, Voreen [27], VisIt [3], and ImageVis3D [9]. For example, ImageVis3D provides a trapezoidal tool for this exploration task in Figure 2 We mimic this user search process by applying image segmentation to recursively divide the histogram into intuitive regions. We show effective discovery of interesting regions by traversing a hierarchy. 


\subsection{Contributions}

We present a visual-data-driven approach to volume data exploration. Users explore a hierarchy to search for regions of interest from coarse to fine.

- We address the information challenge by extracting informative regions using image segmentation on reduced statistics. We mimic user explorations by visually segmenting the 2D histograms. We show these automatic 2D histogram segments wellapproximate meaningful 3D volume segments. These segments fit the shape of the histogram and cover the entire domain. We discuss this segmentation approach in Section 4

- We address the completeness challenge by constructing a complete exploration hierarchy. This hierarchy organizes segments of different sizes from coarse to fine. We progressively visualize the volume dataset by traversing this hierarchy. We show the construction of this hierarchy in Section 5.1

- We assist the exploration by using information-theoretic measures of the volumetric data segments. We evaluate the entropies of the segments and the information gains of the subdivisions. We show how they can assist the exploration in Section 5.2

- We address the semantic challenge by providing intuitive interactions to explore segments at different sizes. Users can effectively identify regions of interest by traversing the hierarchy of segments. This interaction is detailed in Section 5.3

\section{Related Work}

In this paper we show how to interactively explore a volume dataset using a histogram segmentation hierarchy. The two areas of research that are the closest to our work are transfer function design and visual data segmentation, which we overview them next.

\subsection{Transfer Function Design}

Transfer functions directly influence the visualization by assigning optical properties such as color and opacity to voxels. Previous work has devoted a lot of effort on transfer function generation. Pfister et. al. [29] present a survey of different approaches to transfer function design. Traditionally, a transfer function maps voxels to colors and opacity according to a 1D function of the scalar values. Subsequent work has considered multiple attributes and has involved the design of transfer functions using multiple dimensions. Fujishiro et. al. [11] and Zhou et. al. [46] analyze the topology of the scalar field to generate transfer functions. Tzeng et. al. [42] paint on the volume data to design high-dimensional transfer functions. Sereda et. al. [36, 37] compute LH histograms to detect material boundaries and transfer functions. Salama et. al. [34] include abstract semantic attributes to assist in domain-specific visualization design. Kim and Varshney [22] use volumetric saliency to enhance the visualization. Correa and Ma [5, 6] have recently incorporate size and visibility into transfer functions. Ruiz et. al. [33] generate automatic transfer functions based on information divergences.

A popular transfer function attribute is the derivatives of the scalar field, often the gradient of the intensity. Kindlmann and Durkin [23] use the derivatives to show better separation of materials and boundaries. Kniss et. al. [24] use fixed shape widgets to interactively design 2D transfer functions. Roettger et. al. [32] create transfer functions by clustering the $2 \mathrm{D}$ volume histogram according to the spatial connectivity of the volume dataset. Users interact with a $1 \mathrm{D}$ histogram to manipulate the 2D non-parametric segments in Maciejewski et. al.'s system [26]. Instead of the volume histograms, Selver and Güzeliç [35] initialize the transfer function by fitting radial basis functions to the histograms of the image slices in a volume dataset. Most recently, Wang et. al. [44] learn a Gaussian Mixture Model from the volumetric scalar field and use the resulting ellipses to compose transfer functions.

In this paper, we show how to automatically identify segments of interest from the intensity-gradient histograms. We organize these segments from coarse to fine to facilitate the exploration process. Our segments are non-parametric and tightly cover the feature space. Users can interact with these segments directly on the intensity-gradient histograms.

\subsection{Visual Data Segmentation}

Separating 2D images and 3D volumes into meaningful regions is a long-standing and challenging problem. Here we present a small subset of recent results from the vast literature on this topic. Freixenet et. al. [10] survey different approaches to segment 2D images. Mean-shift image segmentation [4] treats the image as probability distribution and finds the segmentation according to local maxima. Graph-based segmentation approaches model the pixels as nodes and the image as a connected graph and then they partition the graph to solve the segmentation problem. Felzenszwalb and Huttenlocher 8 use a boundary predicate and a greedy algorithm to segment images. Sharon et. al. [38] introduce an algebraic multigrid inspired segmentation algorithm.

$3 \mathrm{D}$ volume segmentation is a natural extension to the $2 \mathrm{D}$ image segmentation problem. Huang and Ma [14] apply the region growing technique to volume segmentation. Sherbondy et. al. [39] accelerate volume segmentation with programmable graphics hardware. Segmentation of organs from medical data is a prime application of volume segmentation. Grady et. al. [13] apply random walk segmentation to detect organs from medical volumes. Fuller et. al. [12] use supportvector machines to segment retinal layers. Many systems incorporate user guidance to perform interactive segmentation. Bartz et. al. [1] use a seeded point to segment the bronchi in the lungs. Prassni et. al. [30] incorporate user guidance to minimize the uncertainties in the segmentation. Kniss et. al. [25] use supervised manifold distance to segment volume data. Torsney-Weir et. al. [41] estimate visual responses to guide the search for the image segmentation parameters.

In this paper, we use the popular normalized-cut approach [40] to directly segment the intensity-gradient histogram of a volume data and construct the transfer function. The normalized-cut approach to $2 \mathrm{D}$ image segmentation has been extensively used for a variety of applications and it has been mapped on a variety of architectures including multi-core and many-core GPUs [15].

\section{OVERVIEW}

We segment the intensity-gradient histogram of a volumetric dataset into a hierarchy of regions. We have observed that these regions express meaningful features and boundaries in the $3 \mathrm{D}$ volumes. The resulting hierarchy guides users to interactively explore the dataset. Figure 1 shows an overview of our approach.

Information Challenge: We address the information challenge by segmenting intensity-gradient $2 \mathrm{D}$ histograms of a volumetric dataset into potential regions of interest. The histogram is the most commonly used tool to help in transfer function design. Kniss et. al. [24] have shown that 2D segments in the intensitygradient domain correspond to meaningful 3D regions in the dataset Users recognize these shapes and patterns from the histogram's image and explore the corresponding $3 \mathrm{D}$ regions. We mimic this user behavior by employing the normalized-cut image segmentation to extract such potential regions of interest from a $2 \mathrm{D}$ intensity-gradient histogram.

Completeness Challenge: The normalized-cut segments collectively span the entire intensity-gradient histogram. In order to also cover features of different sizes, we recursively apply the normalizedcut algorithm to obtain a hierarchy of segments. This multilevel hierarchy organizes the segments from coarse to fine. We have found this to be highly usable for interactive exploration.

Semantic Challenge: To address the semantic challenge, we provide interactive exploration with the multilevel segmentation hierarchy. Users traverse through the hierarchy to sift for meaningful features. They can cull away the irrelevant segments and subdivide the relevant segments to explore the details. We evaluate the entropies and information gain in this hierarchy to aid the exploration. These information theoretic measures assist the users in deciding where to 

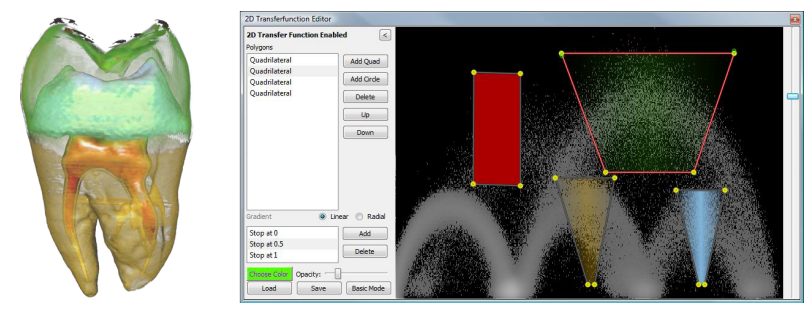

(a)

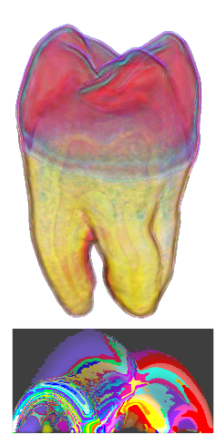

(b)

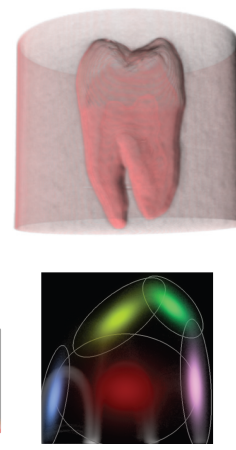

(c)
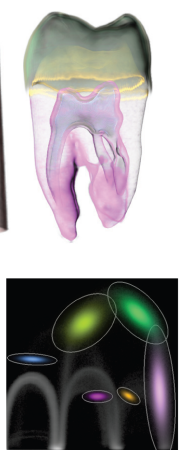

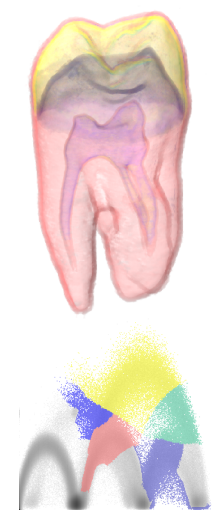

(d)
Fig. 2. Comparison of the Tooth Dataset visualization: (a) shows a user-specified visualization in the ImageVis3D manual using trapezoidal widgets. (b) shows how the spatial transfer function oversegments the histogram into many regions; (c) shows the Gaussian mixture elliptical transfer function. The ellipses do not fully cover the histogram. The left visualization shows the automatic starting configuration. The users can then resize, rotate, and split the ellipses to obtain the right visualization. (d) shows the results from our approach in which we automatically produce a reasonable number of segments. Users can visualize the surfaces by progressively hiding the solid segments, tooth holding material, dentine, and the enamel. Images courtesy of 9932,43

explore. The exploration composes a visualization with features of different sizes.

\subsection{Comparison}

We show a visual comparison of the Tooth dataset in Figure 2 The corresponding segments and histograms in the intensity-gradient domain are shown beneath the rendered image. Figure 2 a) shows a userspecified visualization in ImageVis3D. In Figure 2(b), spatial transfer function [32] connects voxels and histogram pixels in a bottom-up fashion and oversegments the histogram into many regions. Our topdown segmentation is progressive and only divides the histogram into a manageable number of segments.

Region-growing techniques with parametric shapes show limited coverage. Figure 2 b) shows the Gaussian mixture transfer function [43. 44]. Automatic fitting produces the left visualization and requires manual resize, translate, rotate, and split operations on the transfer-function ellipses to produce the visualization on the right. Our visual segmentation approach produces a small number of freeform segments, that tightly fit the histogram and guarantee a complete coverage. We recursively apply the segmentation to also cover features of different sizes.

Figure 2 (c) shows our results. We show the tooth surfaces by identifying and hiding solid segments of tooth holding material, dentine, and the enamel. The key advantages of our approach include:

- Assisting region search by visually segmenting the intensitygradient histogram into collectively exhaustive and mutually exclusive segments.

- The multilevel segmentation hierarchy completely covers features of all sizes.
- Users interacts with a familiar and augmented feature space that is intuitive. No new features are introduced to disrupt the workflow.

\section{Volume Segmentation by Normalized Cut on 2D His- TOGRAMS}

We aim to mimic how users would visually process an intensitygradient histogram. Given a 3D intensity field, we compute its derivative, the gradient, to form a $2 \mathrm{D}$ intensity-gradient histogram. We plot the intensity on the horizontal axis and gradient on the vertical axis. Users locate shapes and patterns from this $2 \mathrm{D}$ histogram to decide how to explore the 3D volume. As shown in Figure 2[a), users use widgets of different shapes to highlight the intensity-gradient histogram and visualize the regions of interest. We aid this tedious process by using image-segmentation algorithms to cut along the shape of this histogram. Previous work [24, 26, 32, 44] has shown that the continuity in the intensity-gradient domain reasonably approximates the spatial continuity in the dataset. We refer our readers to Kniss et. al.'s [24] work, for specific examples of how intensitygradient histogram shapes map to corresponding volume regions in datasets.

Normalized-cut image segmentation [40] divides the histogram into continuous shapes that we seek. It models an image as a graph and finds the best way to partition this graph into $k$ components. Every pixel in the image is considered as a node on the graph. The edge weights, $w(u, v)$, between the nodes, $u$ and $v$, are computed as color and location similarities between the pixels. The closer the pixels, the stronger the edge weight is. The details of the similarity measure we used can be found in [7]. The normalized cut seeks to disconnect the graph, $V$, into components $A, B$ by removing the edges with the least normalized cost. The formulation of the normalized cut is as follows:

$$
\begin{aligned}
\operatorname{cut}(A, B) & =\sum_{u \in A, v \in B} w(u, v) \\
\operatorname{assoc}(A, V) & =\sum_{u \in A, t \in V} w(u, t) \\
\operatorname{Ncut}(A, B) & =\frac{\operatorname{cut}(\mathrm{A}, \mathrm{B})}{\operatorname{assoc}(\mathrm{A}, \mathrm{V})}+\frac{\operatorname{cut}(\mathrm{A}, \mathrm{B})}{\operatorname{assoc}(\mathrm{B}, \mathrm{V})}
\end{aligned}
$$

where, $\operatorname{cut}(\mathrm{A}, \mathrm{B})$ is the total weight of edges connecting components $A$ and $B, \operatorname{assoc}(A, V)$ is the total weight of the edges of $A$ in $V$, and $\operatorname{Ncut}(A, B)$ normalizes cut $(A, B)$ by the edge weights of $A$ and $B$. This normalization favors balanced segments, since small cuts will have a smaller denominator, and therefore, the value of $\operatorname{Ncut}(A, B)$ is increased. Finding the minimum $\operatorname{Ncut}(A, B)$ is a NP-complete problem. This is usually approximated by solving an eigenvalue problem:

$$
\begin{aligned}
& (D-W) y=\lambda D y \\
& d(u)=\sum_{v} w(u, v)
\end{aligned}
$$

where $W$ is the adjacency matrix of the image graph with edge weights $w(u, v), D$ is a diagonal matrix with entries, $d(u)$, and $\lambda$ is an eigenvalue. We can use the resulting eigenvectors, $y$ to partition the graph. Yu and Shi [45] show how to find $k$ partitions by finding $k$ eigenvectors of the eigenvalue problem.

We segment the intensity-gradient histogram using a normalizedcut approach to mimic a semantically meaningful segmentation of the volume dataset. We construct a $2 \mathrm{D}$ histogram image from the volume dataset. We apply the logarithmic function to map the large range of frequencies to grayscale pixels. We trade the $3 \mathrm{D}$ spatial connectivity information for a compact abstraction in the form of a 2D histogram. The size of the histogram representation is independent of the size of the volume; it is only dependent on the bin sizes and precision, which 

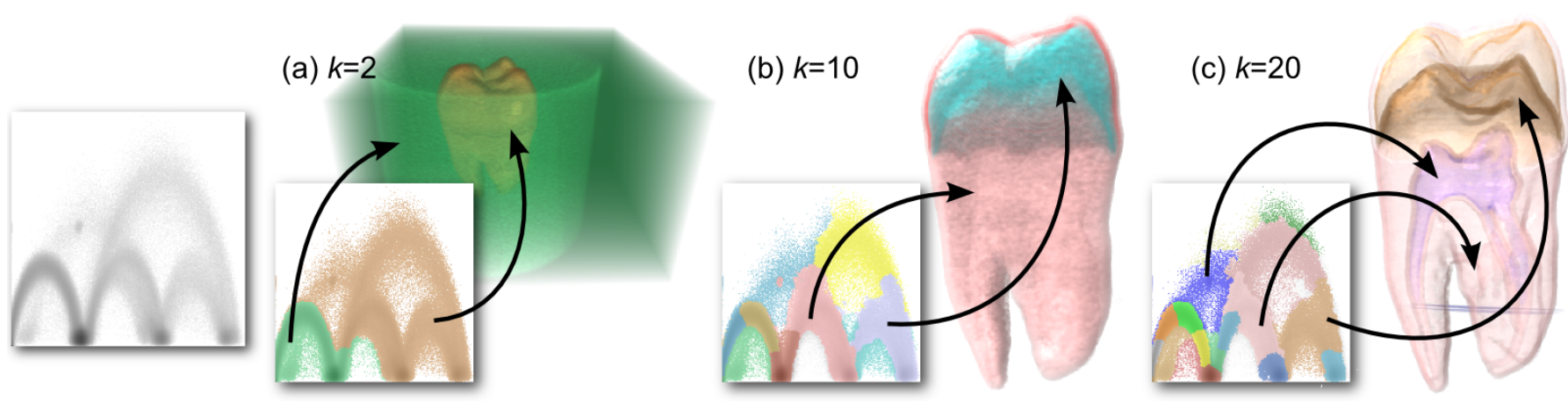

Fig. 3. We show normalized-cut histogram segmentations of the Tooth dataset at different $k$ 's. (a) shows the separation of the tooth and the volume box. (b) shows segments of the crown and root of the tooth. At $k=20$, (c) shows material boundaries that are separated from the solid components.

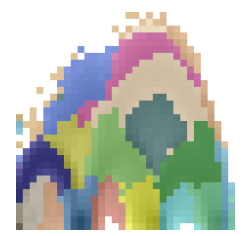

(a) $32 \times 32$

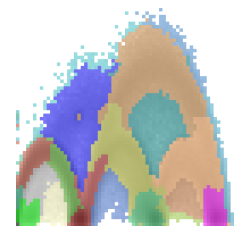

(b) $64 \times 64$

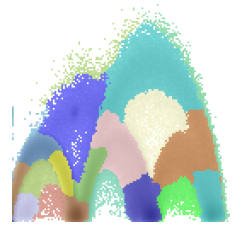

(c) $128 \times 128$

Fig. 4. $k=20$ segmentations of the Tooth's histogram at various resolutions.

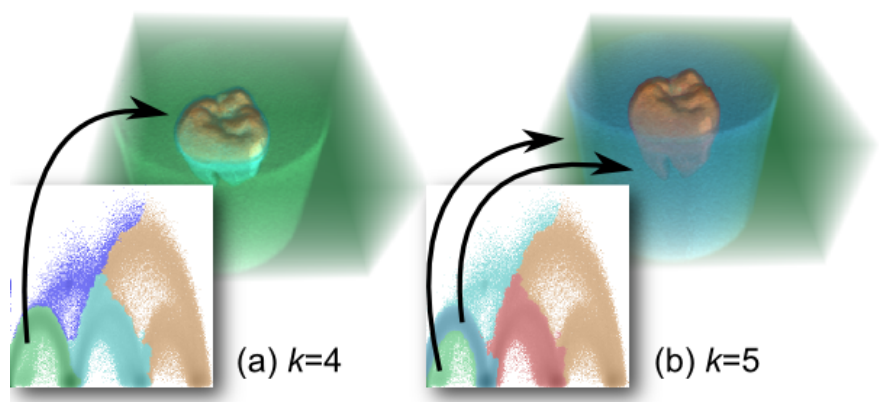

Fig. 5. When we increase $k$ from 4 to 5 , the volume box is segmented into empty space and the cylindrical material that holds the tooth. Although this is a legitimate segmentation, it does not segment the tooth, the region of interest.

can be controlled during the histogram construction. The normalizedcut segmentation procedure divides a 2D histogram into multiple segments. These non-parametric segments fit the histogram tightly as they completely cover the histogram's intensity-gradient domain.

Figure 3 shows example segments of the Tooth dataset and how this normalized-cut approach to the intensity-gradient histogram segmentation results in intuitively meaningful segments. We overlay the segments onto the 2D histograms and show the corresponding 3D segments. The normalized-cut separates the tooth from exterior material at $k=2$. At $k=10$, the segments distinguish solid components and the tooth crown and the root are easily identified. The segments at $k=20$, distinguish material boundaries from the solid components. In our experiments, we construct a histogram from the dataset and store it as a grayscale image. We compute the normalized cut using Cour et. al. [7]'s software. We find $256 \times 256$ is an appropriate resolution and we show approximation of the same histogram at various resolutions in Figure 4

\section{HiERARCHICAL EXPLORATION}

We recursively segment the histogram to form a hierarchy for interactive exploration. A common problem in segmentation is determining the desired number of segments, $k$. Searching for an appropriate $k$ by

repeating the segmentation is a tedious exercise.

Furthermore, as $k$ increases, the new segments may not subdivide any region of interest. For example, Figure 5 shows the segmentation of the Tooth dataset with $k=4$ and $k=5$. We see an additional segment that divides up the box instead of the tooth as $k$ increases from 4 to 5. The newly-divided segments represent the empty space and the material that holds the tooth. Although these are legitimate segments, most users would prefer to segment the tooth.

\subsection{Multilevel Segmentation Hierarchy}

To eliminate the need for a predetermined $k$, we recursively apply normalized cuts to segment the histogram and build a binary hierarchy. This hierarchy lets users to explore the histogram segments from coarse to fine details. Users interactively subdivide and explore selective regions of interest. For example, the users may interactively subdivide the tooth while keeping the box intact. This hierarchy covers the entire intensity-gradient domain with segments of different sizes to ensure an exhaustive exploration.

Any cut through this segmentation hierarchy covers the entire dataset. We can traverse this hierarchy to explore the dataset at varying levels of detail in different regions of the volume. Figure 6 shows how the parent histogram nodes in the hierarchy are subdivided into the children histogram nodes. In this example, we choose to explore the tooth while keeping the segment of tooth-holding materials and space intact at the first level. The second level of the hierarchy shows the segmentation of the solid tooth crown in the third (lower-rightmost) arc. The third level contains segments of the tooth root and the shell of the crown. We can compose a visualization that covers the whole tooth with these segments at different sizes as shown on the right of that figure.

This multilevel segmentation hierarchy provides a mechanism for users to compose visualizations by flexibly combining segments of different sizes. Given the shapes of the histogram segments or corresponding volume regions, users can efficiently decide where to explore and refine. In the next section we show how information-theoretic measures can support in the intuitive segmentation of the hierarchy.

\subsection{Information Content}

We evaluate the information content of a segment or a subdivision to aid the exploration. In addition to the user's intuition and domain knowledge, information content can serve as an auxiliary assistance when traversing the segmentation hierarchy. The use of informationtheoretic measures is increasingly popular in visualization [2]. Kim and $\mathrm{JaJa}[21]$ build information-aware octrees to extract isosurfaces. Jänicke et. al. [17, 18, 19] apply information theoretic measures to visualize flow. Ruiz et. al. [33] compose automatic transfer functions based on information divergence.

We compute the entropy at each segment and evaluate the information gain at each subdivision of the segmentation hierarchy. Our goal is to lead users to explore segments that are more informative. To assist user exploration, we characterize the segments and the subdivision 


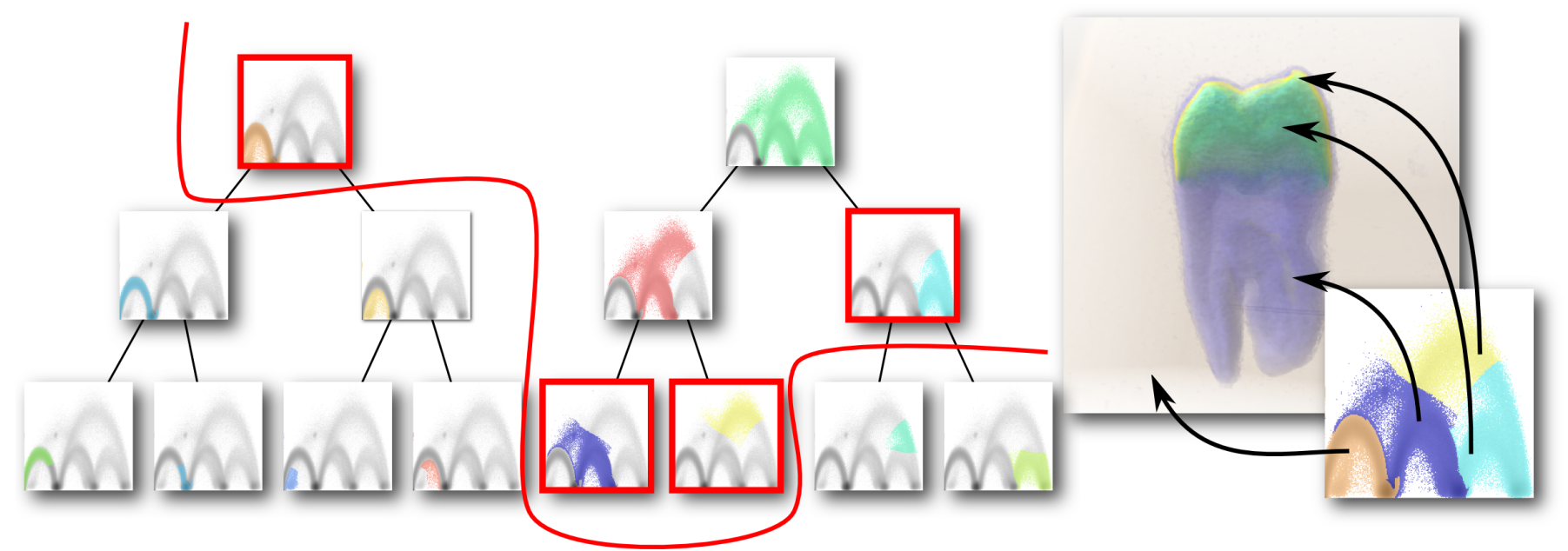

Fig. 6. A multilevel segmentation hierarchy enables users to compose visualizations with segments of different sizes. Assembling the red-framed segments produces the visualization on the right. Similar to a level-of-details hierarchy, a cut in this hierarchy covers the entire dataset.

with two different information theoretic measures: (a) Entropy, and (b) Information Gain.

Segment Entropy: We use entropy to characterize the complexity of a segment. For each segment (the nodes in the hierarchy), we extract sub-volumes, $V$, by grouping the voxels with the corresponding intensities and gradient values. We compute the entropy in the sub-volumes, $H(V)$, as follows:

$$
H(V)=-\sum_{i} p\left(v_{i}\right) \lg _{2} p\left(v_{i}\right)
$$

where $v_{i}$ is a voxel in $V, p\left(v_{i}\right)$ is the probability of $v_{i} . p\left(v_{i}\right)$ can be computed by analyzing how many voxels have the same intensity as $v_{i}$ in $V$. This classic Shannon entropy measures how many bits are required to encode $V$. A high number of bits required represents a higher complexity.

In Figure 7(a), we color the segments of the Tooth dataset according to their entropy. It shows that the tooth contains a higher entropy than the empty space and tooth-holding material. However, when we further subdivide the tooth in Figure 7 (b) the entropies of different components start converging and it becomes less clear which segment should be further explored. To address this we next discuss how to evaluate the information gain.

Information Gain: We use information gain to evaluate the effect of subdividing a segment. The information gain is the reduction in entropy after a subdivision. This measure has been widely used in building decision trees [31], where the attribute with the highest information gain subdivides the training data. We compute this by subtracting the entropy of the children nodes from the parent node:

$$
G(V)=\left(H(V)-\sum_{j} \frac{\left|V_{j}\right|}{|V|} H\left(V_{j}\right)\right) / H(V)
$$

where $V_{j}$ are the sub-volumes of $V$. We normalize this gain by the entropy before the subdivision. Figure 7 b) shows how information gain can distinguish segments with similar entropy. In this case subdividing the high information gain segment separates the dentine boundary from the enamel crown. The entropy reduction highlights potential structures, even if the complexity of the segments are similar. When the entropy reduction is low, it means that the subdivision does not sufficiently reduce the complexity of the segment. However, if the entropy reduction is high, it suggests that the complexity is lowered in the sub-segments, which is likely to happen as a result of separation of two meaningfully different structures into the two sub-segments. While, the noise in the dataset may affect the computation of the true entropy, the relative nature of the information-gain measure should reduce this noise effect.

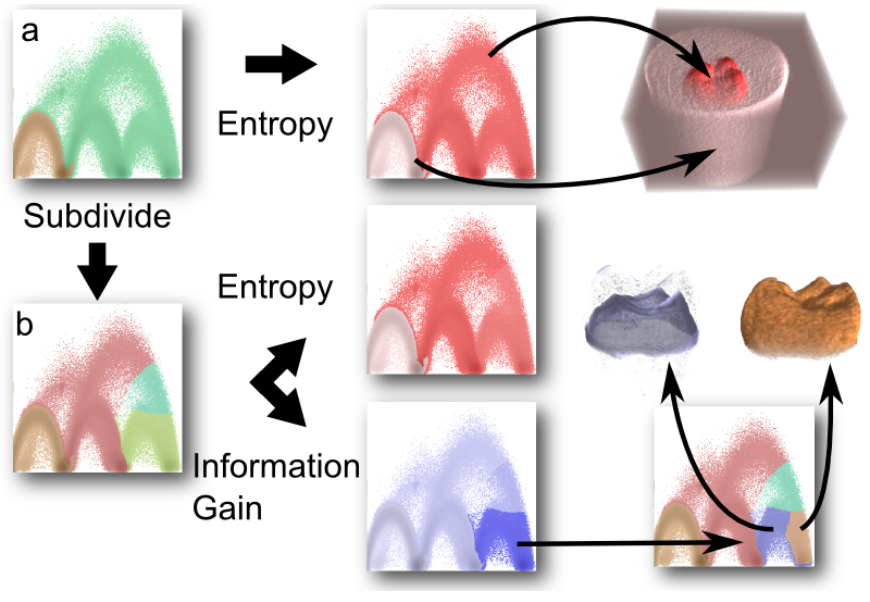

Fig. 7. (a) shows the tooth segment has a higher entropy (in red) than the empty space and tooth-holding material. Further subdivision the tooth in (b) results in segments with converging entropies. In an alternate approach, we evaluate the information gain on those segments. The subdivision with the highest information gain (in blue) separates the dentine boundary from the enamel crown.

\subsection{Interactive Exploration}

The users interact with segments at different levels of the hierarchy and compose visualizations on the intensity-gradient histogram. Interactive and multilevel exploratory approaches have been shown effective on large and complex datasets, such as, lengthy molecular simulations [28] and very large gigapixel images [16]. This interactive approach allows users to incorporate their knowledge in the exploration process. The main interactions in this procedure are subdividing or hiding a segment. Users inspect the segments and decide the subsequent exploration process. Hand editing of segments, such as, manual translation, rotation, resizing, or reshaping of segments is not required in the results presented in this work.

We present the dataset in a bi-segment configuration to start the exploration. Similar to other related work, we overlay the segments on the intensity-gradient histogram for the users to relate to the volume regions. By default, we color the segments using a randomly-generated color map. Clicking on any segment subdivides it into two components. Users can visualize the entropy or information gain using a color-coded visualization with an appropriate key press. Users may also choose to change the colors and opacities of each segment. These interactions allow a user to inspect and focus on regions of interest. 


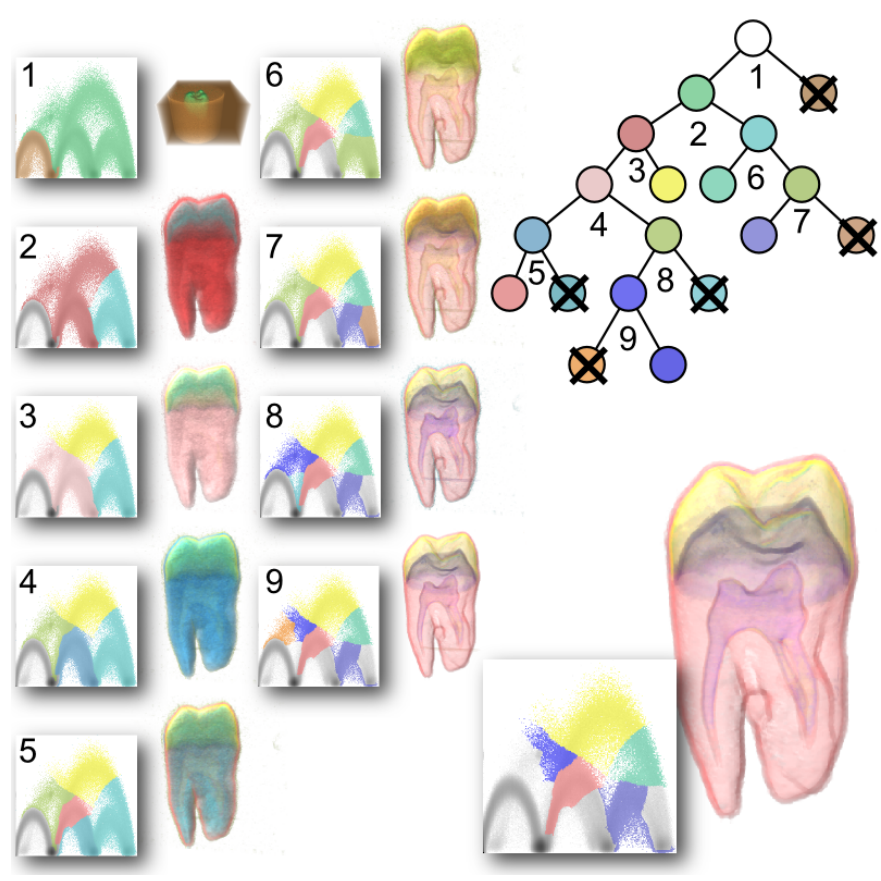

Fig. 8. This shows an example of the interactive exploration process. The hierarchy on the right shows the subdivision steps. The left shows the corresponding visualizations and histogram segments at different steps. The process is detailed in Section 5.3

We also provide shortcuts to clear the opacity of any segment to allow the user to easily cull away any segment.

We show how to exhaustively explore the Tooth dataset and compose a boundary visualization similar to the user-specified visualization in Figure 2 a). The supplementary video shows this visualization can be constructed in less than a minute. Figure 8 presents an example of the interactive exploration process. The hierarchy on the top right shows the subdivision and the numbers indicate the order of subdivision. The corresponding histograms and visualizations are shown on the left. The colors of the nodes are the colors of the corresponding segments. The crossed-out leaves indicate that we decided to cullaway those segments by setting the opacity to zero. Their subtrees are not further explored.

The exploration starts with the full dataset. The initial segments in step 1 divide the tooth from the rest of data, the first (lower-leftmost) arc in the histogram. We focus on the tooth from step 2 onwards. Step 2 shows the crown and the root. Step 3 segments the shell of the crown in yellow. Step 4 shows the second (lower-middle) arc is the root of the tooth and separates it from noise (this is best seen in the video). We first select the root and then remove the noise. Step 5 divides the root into the solid blob-like dentine component and its boundary. We keep the dentine boundary and move on to the tooth crown represented by the third (lower-rightmost) arc in step 6. Step 7 divides the arc, the left half shows the boundary between the enamel and the dentine and the right half shows the solid enamel component. We show the boundary by hiding the solid component and move on to explore the last noise-like segment between the first (lower-leftmost) and second (lower-middle) arcs in step 7. In step 8, the lower half of the noise-like component shows the noise, however the top half shows the boundary of the pulp along with a small piece of the tooth-holding material at the back. We hide the noise, then further segment the pulp boundary in step 9. This allows us to remove the tooth-holding material and arrive at our final visualization.

\section{Results}

We apply this exploration approach to visualize a variety of volume datasets:
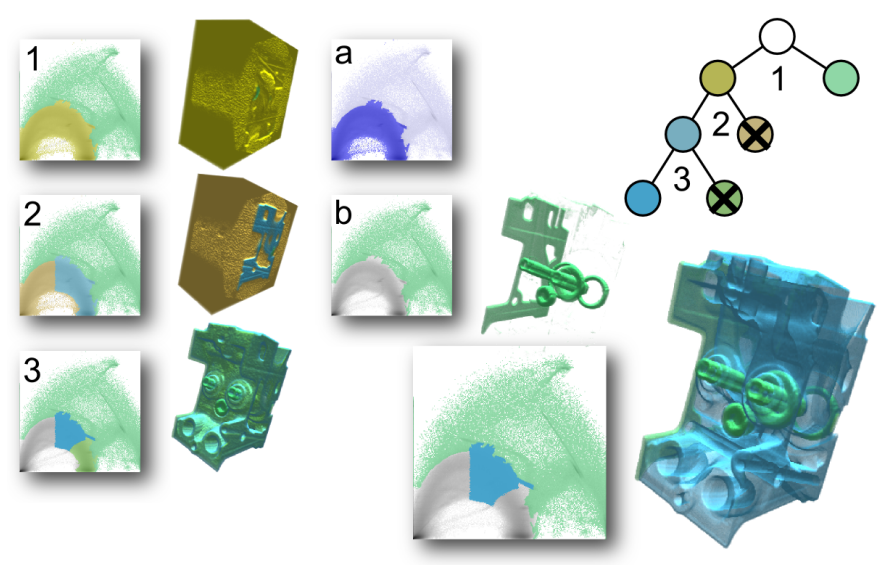

Fig. 9. Visualization of the Engine dataset: in (a) the solid arc region shows a high information gain. We subdivide this arc to separate the engine block from the space and the noise and visualize it along with the internal structures shown in (b).

1. Engine

2. Foot

3. Visible Human Male Head

4. Tomato

5. Hurricane Isabel

Implementation and Experimental Setup: We compute a six-level normalized-cut hierarchy for each histogram by using Cour et. al.'s [7] normalized cut software in Matlab. We only store the finest level of the segmentation and access the rest according to a binary-tree order. We visualize the segments as freeform components on a 2D intensity-gradient transfer function. We implement our application with Qt and the NVIDIA CUDA SDK volume rendering example. The preprocessing in Matlab takes about 15 seconds per histogram. This preprocessing can be avoided completely if we can perform normalized cut with an accelerated eigensolver at exploration time as discussed in Section 7. We performed all experiments on a Linux workstation with an Intel Xeon 5140 and a NVIDIA GeForce 260GTX GPU.

For each dataset, we show a visualization and the corresponding exploration hierarchy. The numbering shows the order of the subdivision steps along the hierarchy. We illustrate the key steps and their corresponding visualizations on the left side of each figure. We also highlight segments corresponding to interesting structures of the datasets. The supplementary video shows the interactive composition process. Our results show our approach follows human intuition in segmenting histograms into meaningful components.

Engine: The engine block's histogram shows a distinctive arc-like region that represents the main engine block and the space around it. The segmentation separates this solid arc region from the other scattered arc with high intensity and gradient in the histogram. The high intensity and high gradient arc represents the internal structures and is shown in Figure 9(b). Figure 9 a) shows the arc region that represents the space and the engine block regions shows a high information gain. We subdivide the arc to separate the space and noise from the engine block. We then obtain the surface of the engine block by subdividing another level. The final visualization shows the engine block surface and the internal structures in Figure 9

Foot: Figure 10 shows the visualization of the Foot dataset. Although the histogram in this foot dataset does not exhibit any distinctive arc shapes, our visual segmentation technique can still divide the histogram into meaningful segments. Step 1 separates the bones from the rest. We subdivide the box of spaces and the flesh in steps 2-5, to obtain segments corresponding to the joints, the flesh, and the skin. Figure 10 (b) shows the visualization of the bones and the joints. We compose the visualization with the bones, the joints, and the skin. 


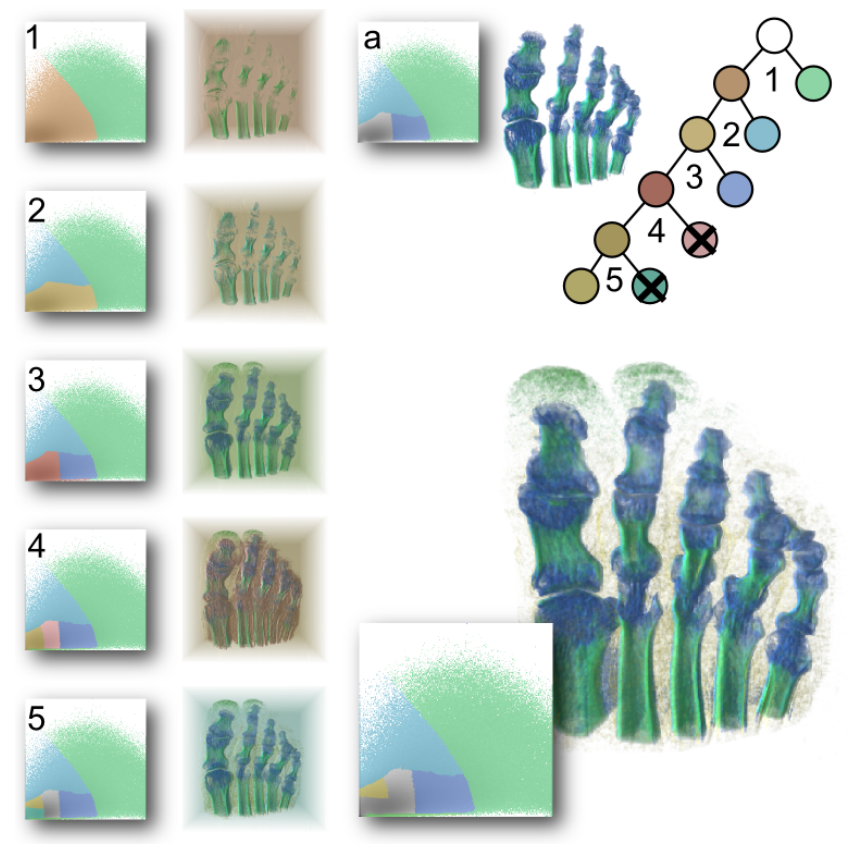

Fig. 10. Visualization of the Foot dataset. Each component of the foot, such as, the bone (Step 1), the joints (Step 3), and the flesh (Step 4) are shown along the composition hierarchy. (a) shows the separated bones and joints.

Tomato: We distinguish different parts of a tomato in Figure 11 The red low intensity and high gradient region is the skin in Figure 11 (c). The blue locular cavities in Figure 11 (b) are characterized by high intensity and high gradient. Notice the yellow collumella in Figure 11 (a) is at the center of the histogram, whose segmentation would not have been possible with an intensity-only 1D histogram.

Visible Human Male Head: We present the head of the visible human male dataset as an example in Figure 12 Similar to the Foot dataset, Step 1 shows the bones. Step 2 shows the teeth. Steps 3, 4, and 5 divide the low-intensity arc region progressively and show the flesh (step 4) and the skin (step 5). Step 6 divides the low-intensity and highgradient regions into the sinus and some noise. We remove the noise and show the bone, skin, teeth, and sinus in the final visualization.

Hurricane Isabel: We visualize the pressure field of the hurricane Isabel dataset. This is a continuous scalar field with no abrupt boundaries and is different from the previous examples with clear material changes. Figure 13 shows the exploration hierarchy of the hurricane. Step 1 separates the land and the atmospheric regions. Step 2 segments the hurricane eye from the atmospheric region. The rainbands from the eyewall structures of the hurricane are separated in Step 3. By inspecting the entropy of the segments in (c), we decide to switch our focus onto the eye of the hurricane. Steps 4 and 5 show regions of different pressures in the hurricane eye. We compose the final visualization with the hurricane eye segments, the eyewall and the rainbands. Figure 13 a) and (b) show the separated eyewall and the rainbands.

\section{Conclusions and Future Work}

In this paper we present a hierarchy of normalized-cut-assisted visual segmentation of an intensity-gradient histogram to assist in the volume exploration process. In contrast with existing approaches in Figure 2 b) and (c), our top-down segmentation approach produces fewer segments and a superior coverage. Our visual approach also well approximates user-specified visualizations in Figure 2(a). We address the information challenge by using a visual segmentation of intensitygradient histograms to locate various meaningful volumetric segments. These segments completely cover the intensity-gradient domain in the image space and identify features of different sizes. We show that any

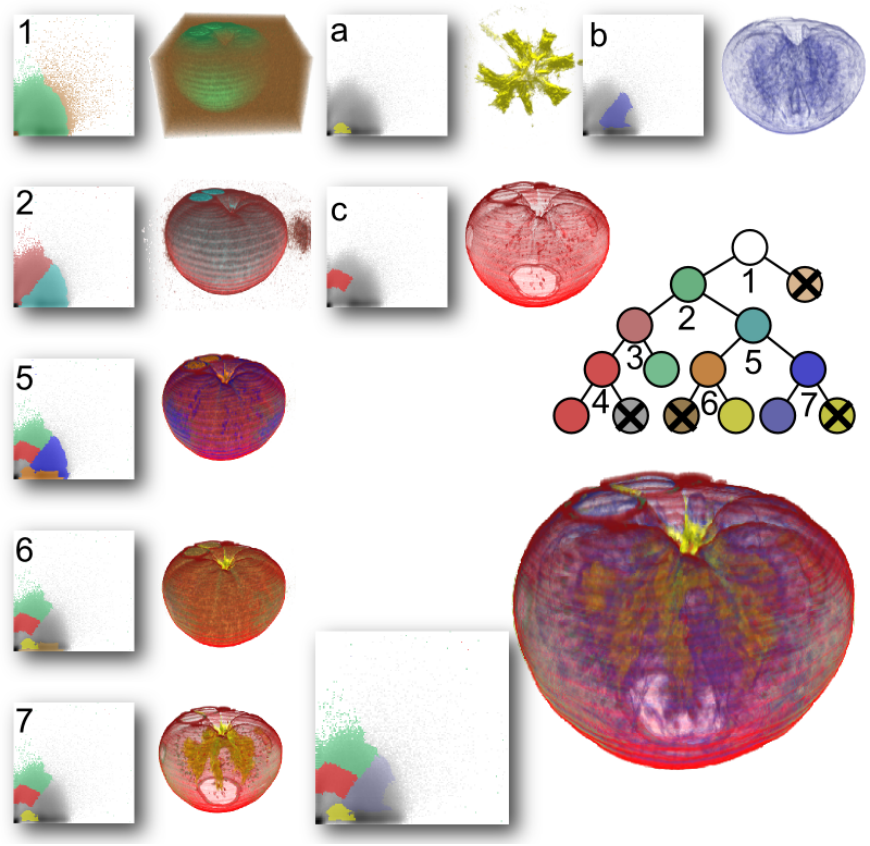

Fig. 11. Visualization of the Tomato dataset. Steps 1-4 lead to the skin segment in (c). Steps 5-6 show the columella and placenta in (a) The locular cavity is shown in (b). The visualization shows the skin, locular cavity, columella, and placenta of the tomato.

cut in the segmentation hierarchy covers the entire dataset. Exploring along this hierarchy addresses the completeness challenge. We assist the volumetric data exploration process by using information-theoretic measures. The users can identify meaningful components and material boundaries through a concise interactive exploration procedure. These interactions address the semantic challenge by allowing users to adaptively explore the dataset and associate their knowledge with the corresponding data segments.

We believe our hierarchical approach naturally extends to larger datasets. The key is to perform the normalized-cut segmentation ondemand. This is critical for visualizing a large dataset because it can limit the subdivisions and segmentation to only the necessary ones. The hierarchical nature ensures that the complexity of subdividing volumes does not grow. Another possible avenue of advancement is to study other, more sophisticated measures of information content for the volumetric subdivisions. The general entropy characterizes the worst-case uncertainty. We believe this may be improved by considering the spatial structure in the volume datasets.

\section{ACKNOWLEDGMENTS}

We thank the anonymous reviewers for their constructive comments and suggestions that have greatly improved the presentation of this work. This work has been supported in part by the NSF grants: CCF 05-41120, CMMI 08-35572, CNS 09-59979 and the NVIDIA CUDA Center of Excellence. Any opinions, findings, conclusions, or recommendations expressed in this article are those of the authors and do not necessarily reflect the views of the research sponsors.

\section{REFERENCES}

[1] D. Bartz, D. Mayer, J. Fischer, S. Ley, A. Rio, S. Thust, C. P. Heussel, H. U. Kauczor, and W. Straßer. Hybrid segmentation and exploration of the human lungs. In IEEE Visualization, page 24, 2003.

[2] M. Chen and H. Jänicke. An information-theoretic framework for visualization. IEEE Transactions on Visualization and Computer Graphics, 16:1206-1215, November 2010. doi:10.1109/TVCG.2010.132

[3] H. Childs, E. Brugger, K. Bonnell, J. Meredith, M. Miller, B. Whitlock, and N. Max. A contract based system for large data visualization. In 


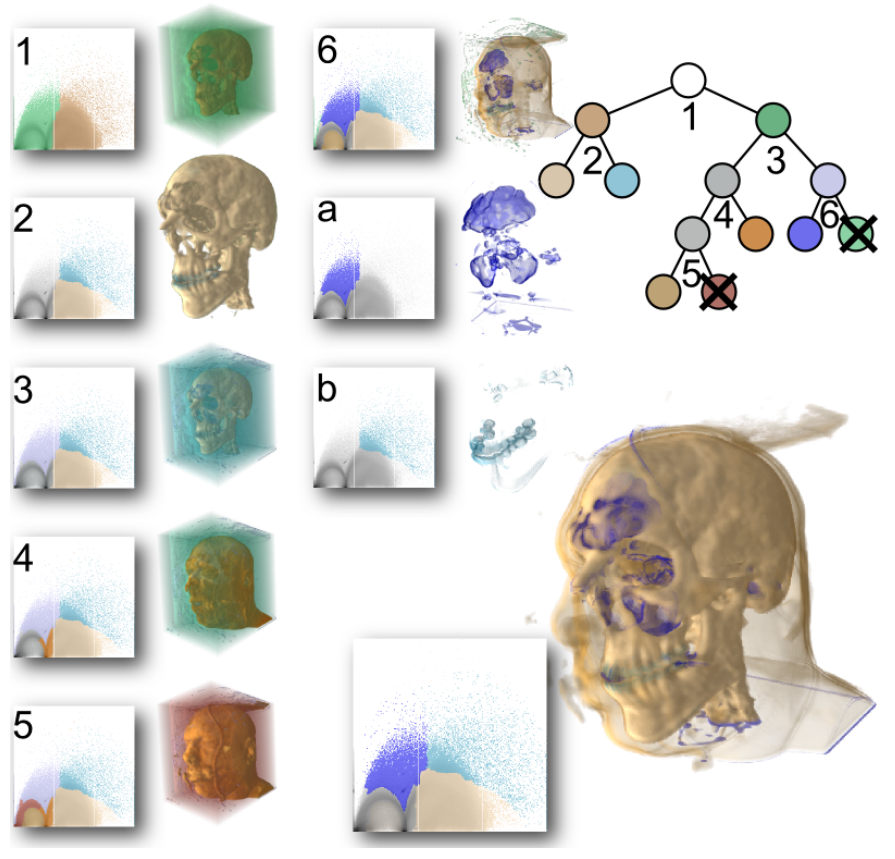

Fig. 12. Visualization of the Visible Human Male Head dataset. The hierarchy progressively (2-6) reveals the bone, teeth, flesh, skin, and sinus. (a) shows the sinus segment and (b) shows the teeth segment. We compose the visualization with the bone, teeth, skin and sinus.

IEEE Visualization, pages 191 - 198, 2005. doi:10.1109/VISUAL. 2005.1532795

[4] D. Comaniciu and P. Meer. Mean shift: a robust approach toward feature space analysis. IEEE Transactions on Pattern Analysis and Machine Intelligence, 24(5):603-619, 2002. doi:10.1109/34.1000236

[5] C. Correa and K.-L. Ma. Size-based transfer functions: A new volume exploration technique. IEEE Transactions on Visualization and Computer Graphics, 14(6):1380-1387, 2008. doi:10.1109/TVCG. 2008.162

[6] C. D. Correa and K.-L. Ma. Visibility histograms and visibility-driven transfer functions. IEEE Transactions on Visualization and Computer Graphics, 17:192-204, 2011. doi:10.1109/TVCG.2010.35

[7] T. Cour, F. Benezit, and J. Shi. Spectral segmentation with multiscale graph decomposition. In Computer Vision and Pattern Recognition, pages 1124 -1131, 2005. doi:10.1109/CVPR.2005.332

[8] P. Felzenszwalb and D. Huttenlocher. Efficient graph-based image segmentation. International Journal of Computer Vision, 59(2):167-181, 2004.

[9] T. Fogal and J. Krüger. Tuvok, an architecture for large scale volume rendering. In International Workshop on Vision, Modeling, and Visualization, 2010.

[10] J. Freixenet, X. Muñoz, D. Raba, J. Martí, and X. Cufí. Yet another survey on image segmentation: Region and boundary information integration. In European Conference on Computer Vision, pages 408-422, 2002.

[11] I. Fujishiro, T. Azuma, and Y. Takeshima. Automating transfer function design for comprehensible volume rendering based on 3D field topology analysis (case study). In IEEE Visualization, pages 467-470, 1999.

[12] A. R. Fuller, R. J. Zawadzki, S. Choi, D. F. Wiley, J. S. Werner, and B. Hamann. Segmentation of three-dimensional retinal image data. IEEE Transactions on Visualization and Computer Graphics, 13(6):17191726, 2007. doi:10.1109/TVCG.2007.70590

[13] L. Grady, T. Schiwietz, S. Aharon, and R. Westermann. Random walks for interactive organ segmentation in two and three dimensions: Implementation and validation. In MICCAI, pages 773-780, 2005.

[14] R. Huang and K.-L. Ma. Rgvis: Region growing based techniques for volume visualization. In Pacific Graphics, pages 355-363, 2003

[15] M. Hussein, A. Varshney, and L. Davis. On implementing graph cuts on CUDA. In First workshop on General Purpose Processing on Graphics Processing Units, 2007.

[16] C. Y. Ip and A. Varshney. Saliency-assisted navigation of very large land-

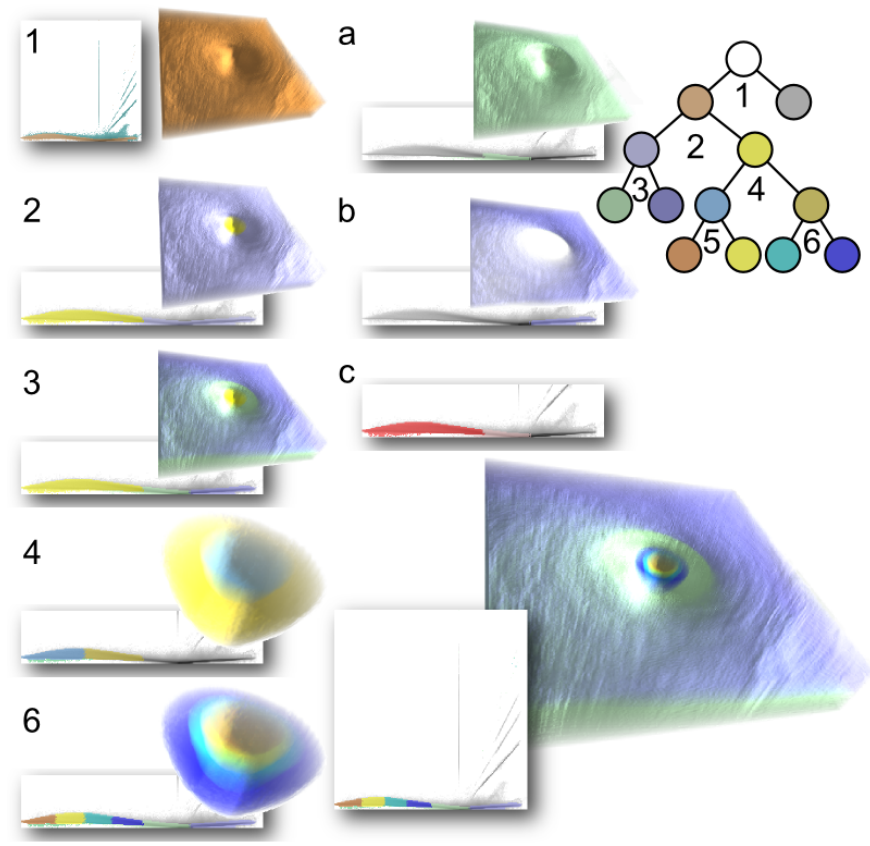

Fig. 13. Visualization of the pressure field of the Hurricane Isabel dataset. We magnify the bottom part of the histogram in steps 2-6, (a), (b), and (c) to better show the segments. Steps 1-3 divide the hurricane into its major components: the eye, the eyewall, and the rainbands. (c) shows the entropy of the Hurricane eye is higher than the other segments after step 3. We focus on the hurricane eye in steps 4-6. (a) shows the eyewall segment and (b) shows the rainbands segment.

scape images. IEEE Transactions on Visualization and Computer Graphics, 17(12):1737 -1746, 2011. doi:10.1109/TVCG.2011.231

[17] H. Jänicke, M. Bottinger, U. Mikolajewicz, and G. Scheuermann. Visual exploration of climate variability changes using wavelet analysis. IEEE Transactions on Visualization and Computer Graphics, 15(6):13751382, 2009.

[18] H. Jänicke and G. Scheuermann. Measuring complexity in Lagrangian and Eulerian flow descriptions. Computer Graphics Forum, 29(6):17831794,2010

[19] H. Jänicke and G. Scheuermann. Visual analysis of flow features using information theory. IEEE Computer Graphics \& Applications, 30:40-49, 2010. doi:10.1109/MCG.2010.17

[20] C. Johnson. Top scientific visualization research problems. IEEE Computer Graphics \& Applications, 24(4):13 - 17, 2004. doi:10.1109/ MCG.2004.20

[21] J. Kim and J. JaJa. Information-aware $2^{n}$-tree for efficient out-of-core indexing of very large multidimensional volumetric data. In International Conference on Scientific and Statistical Database Management, July 2007. doi:10.1109/SSDBM.2007.15

[22] Y. Kim and A. Varshney. Saliency-guided enhancement for volume visualization. IEEE Transactions on Visualization and Computer Graphics, 12(5):925-932, 2006.

[23] G. L. Kindlmann and J. W. Durkin. Semi-automatic generation of transfer functions for direct volume rendering. In IEEE Symposium on Volume Visualization, pages 79-86, 1998.

[24] J. Kniss, G. Kindlmann, and C. Hansen. Multidimensional transfer functions for interactive volume rendering. IEEE Transactions on Visualization and Computer Graphics, 8:270-285, 2002. doi:10.1109/ TVCG.2002.1021579

[25] J. Kniss and G. Wang. Supervised manifold distance segmentation. IEEE Transactions on Visualization and Computer Graphics, 17(11):1637 1649, 2011. doi:10.1109/TVCG.2010.120

[26] R. Maciejewski, I. Woo, W. Chen, and D. Ebert. Structuring feature space: A non-parametric method for volumetric transfer function generation. IEEE Transactions on Visualization and Computer Graphics, 15:1473-1480, 2009. doi:10.1109/TVCG.2009.185

[27] J. Meyer-Spradow, T. Ropinski, J. Mensmann, and K. Hinrichs. Voreen: 
A rapid-prototyping environment for ray-casting-based volume visualizations. IEEE Computer Graphics \& Applications, 29(6):6-13, 2009. doi:10.1109/MCG.2009.130

[28] R. Patro, C. Y. Ip, S. Bista, S. Cho, D. Thirumalai, and A. Varshney. MDMap: A system for data-driven layout and exploration of molecular dynamics simulations. In BioVis, pages $111-118,2011$. doi: 10.1109/BioVis.2011.6094055

[29] H. Pfister, B. Lorensen, C. Bajaj, G. Kindlmann, W. Schroeder, L. S. Avila, K. Martin, R. Machiraju, and J. Lee. The transfer function bakeoff. IEEE Computer Graphics \& Applications, 21:16-22, 2001. doi: $10.1109 / 38.920623$

[30] J.-S. Prassni, T. Ropinski, and K. Hinrichs. Uncertainty-aware guided volume segmentation. IEEE Transactions on Visualization and Computer Graphics, 16(6):1358 -1365, 2010. doi:10.1109/TVCG.2010. 208

[31] J. R. Quinlan. C4.5: programs for machine learning. Morgan kaufmann, 1993.

[32] S. Roettger, M. Bauer, and M. Stamminger. Spatialized transfer functions. In EuroVis, pages 271-278, 2005.

[33] M. Ruiz, A. Bardera, I. Boada, I. Viola, M. Feixas, and M. Sbert. Automatic transfer functions based on informational divergence. IEEE Transactions on Visualization and Computer Graphics, 17(12):1932-1941, 2011. doi:10.1109/TVCG.2011.173

[34] C. R. Salama, M. Keller, and P. Kohlmann. High-level user interfaces for transfer function design with semantics. IEEE Transactions on Visualization and Computer Graphics, 12(5):1021-1028, 2006.

[35] M. A. Selver and C. Güzeliç. Semiautomatic transfer function initialization for abdominal visualization using self-generating hierarchical radial basis function networks. IEEE Transactions on Visualization and Computer Graphics, 15(3):395-409, 2009.

[36] P. Šereda, A. Bartroli, I. Serlie, and F. Gerritsen. Visualization of boundaries in volumetric data sets using LH histograms. IEEE Transactions on Visualization and Computer Graphics, 12(2):208-218, 2006.

[37] P. Šereda, A. Vilanova, and F. Gerritsen. Automating transfer function design for volume rendering using hierarchical clustering of material boundaries. In EuroVis, pages 243-250, 2006.

[38] E. Sharon, M. Galun, D. Sharon, R. Basri, and A. Brandt. Hierarchy and adaptivity in segmenting visual scenes. NATURE, 442(7104):810, 2006.

[39] A. Sherbondy, M. Houston, and S. Napel. Fast volume segmentation with simultaneous visualization using programmable graphics hardware. In IEEE Visualization, page 23, 2003.

[40] J. Shi and J. Malik. Normalized cuts and image segmentation. IEEE Transactions on Pattern Analysis and Machine Intelligence, 22(8):888905, 2000. doi:10.1109/34.868688

[41] T. Torsney-Weir, A. Saad, T. Moller, H. Hege, B. Weber, J. Verbavatz, and S. Bergner. Tuner: Principled parameter finding for image segmentation algorithms using visual response surface exploration. IEEE Transactions on Visualization and Computer Graphics, 17(12):1892 -1901, 2011. doi:10.1109/TVCG.2011.248

[42] F.-Y. Tzeng, E. B. Lum, and K.-L. Ma. An intelligent system approach to higher-dimensional classification of volume data. IEEE Transactions on Visualization and Computer Graphics, 11:273-284, 2005. doi:10. 1109/TVCG.2005.38

[43] Y. Wang, W. Chen, G. Shan, T. Dong, , and X. Chi. Volume exploration using ellipsoidal Gaussian transfer functions. In PacificVis, pages 25-32, 2010. doi:10.1109/PACIFICVIS.2010.5429612

[44] Y. Wang, W. Chen, J. Zhang, T. Dong, G. Shan, and X. Chi. Efficient volume exploration using the Gaussian mixture model. IEEE Transactions on Visualization and Computer Graphics, 17(11):1560-1573, 2011. doi:10.1109/TVCG.2011.97

[45] S. X. Yu and J. Shi. Multiclass spectral clustering. In International Conference on Computer Vision, pages 313-319, 2003. doi:10.1109/ ICCV.2003.1238361

[46] J. Zhou and M. Takatsuka. Automatic transfer function generation using contour tree controlled residue flow model and color harmonics. IEEE Transactions on Visualization and Computer Graphics, 15(6):1481 $-1488,2009$. doi:10.1109/TVCG.2009.120 